\title{
ARTICLE
}

Infectious medicine, virology

\section{Cancer increases risk of in-hospital death from COVID-19 in persons $<65$ years and those not in complete remission}

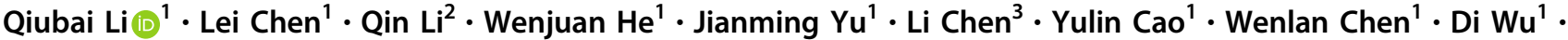

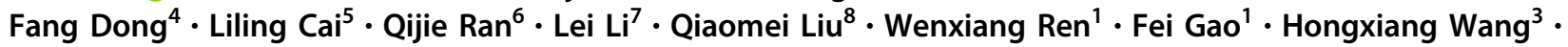 \\ Zhichao Chen ${ }^{1} \cdot$ Robert Peter Gale ${ }^{9} \cdot$ Yu Hu (i) ${ }^{1}$
}

Received: 20 June 2020 / Revised: 25 June 2020 / Accepted: 9 July 2020 / Published online: 20 July 2020

(c) The Author(s), under exclusive licence to Springer Nature Limited 2020

\begin{abstract}
The impact of cancer on outcome of persons with coronavirus disease 2019 (COVID-19) after infection with acute respiratory syndrome coronavirus-2 (SARS-CoV-2) is controversial. We studied 1859 subjects with COVID-19 from seven centers in Wuhan, China, 65 of whom had cancer. We found having cancer was an independent risk factor for in-hospital death from COVID-19 in persons $<65$ years (hazard ratio [HR] $=2.45,95 \%$ confidence interval $[\mathrm{CI}], 1.04,5.76 ; P=0.041)$ but not in those $\geq 65$ years $(\mathrm{HR}=1.12[0.56,2.24] ; P=0.740)$. It was also more common in those not in complete remission. Risks of in-hospital death were similar in subjects with solid cancers and those with hematological cancers. These data may help predict outcomes of persons with cancer and COVID-19.
\end{abstract}

\section{Introduction}

The impact of cancer on outcome of persons developing coronavirus disease 2019 (COVID-19) after infection with acute respiratory syndrome coronavirus-2 (SARSCoV-2) is controversial [1-12]. Several studies reported

These authors contributed equally: Qiubai Li, Lei Chen, Qin Li

Supplementary information The online version of this article (https:// doi.org/10.1038/s41375-020-0986-7) contains supplementary material, which is available to authorized users.

Qiubai Li

qiubaili@hust.edu.cn

$\triangle \mathrm{Yu} \mathrm{Hu}$

dr_huyu@126.com

1 Institute of Hematology, Union Hospital, Tongji Medical College, Huazhong University of Science and Technology, Wuhan 430022, China

2 Department of Vascular Surgery, Union Hospital, Tongji Medical College, Huazhong University of Science and Technology, Wuhan 430022, China

3 Department of Hematology, Wuhan Central Hospital Tongji Medical College, Huazhong University of Science and Technology, Wuhan, China an increased risk of death $[1-3,5-9,13-18]$, others not [19-26]. We studied 1859 subjects with COVID-19 from seven centers in Wuhan, China, 65 of whom also had cancer. We found cancer was an independent risk factor for in-hospital death from COVID-19 in subjects $<65$ but not $\geq 65$ years. Subjects with cancer not in complete remission also had an increased risk of death from COVID-19. Risks of death were similar in subjects with solid cancers and those with hematological cancers. These data may help predict outcomes of persons with cancer and COVID-19.

4 Intensive Care Units of Wuhan Third Hospital, Tongren Hospital of Wuhan University, Wuhan, China

5 Department of Urology Surgery, Union Hospital, Tongji Medical College, Huazhong University of Science and Technology, Wuhan 430022, China

6 Department of Hematology, General Hospital of Central Theater Command, PLA, Wuhan 300700, China

7 Wuhan Jin-Yin-Tan Hospital, Wuhan, China

8 Medical Records Statistics Department of Wuhan Third Hospital, Tongren Hospital of Wuhan University, Wuhan, China

9 Center for Hematology Research, Department of Immunology and Inflammation, Imperial College London, London, UK 


\section{Methods}

\section{Study design and participants}

Between 20 January and 4 April 2020 we studied 1859 consecutive subjects with COVID-19 treated at seven centers of five hospitals of Union Hospital (main part, Union West Hospital and Union Tumor Hospital), Wuhan Central Hospital, General Hospital of Central Theater Command, PLA and Wuhan Jinyintan Hospital. These hospitals were urgently reconstructed by the Chinese government as the designated hospitals and centers for COVID-19 therapy.

Subjects were tested for SARS-CoV-2-infection by qRTPCR of nasal and pharyngeal swabs and/or blood test for anti-SARS-CoV-2 IgG/IgM antibodies using a colloidalgold-based 2019-nCoV IgG/IgM Detection Kit (Nanjing Vazyme Medical Technology, Nanjing, China) using criteria of the Novel Coronavirus Pneumonia Diagnosis and Treatment Program of the National Health Commission of China (7th version) [27] and World Health Organization (WHO) interim guidance [28]. COVID-19 was diagnosed in neighborhood clinics or Fangcang Hospital before admission to the designated hospitals or in designated hospitals after admission [29]. Severity of COVID-19 was graded as described [2]. Subjects were treated as reported [30, 31] or according to Chinese government guidelines. The study was approved by the Ethics Committees of Union Hospital (2020-0095) and of Wuhan Central Hospital (2020-007). Requirement for informed consent was waived by the Ethics Committees.

\section{Data collection}

Electronic medical records (EMRs) of subjects including epidemiological, demographic, and laboratory data were reviewed. Outcomes included: (1) death; (2) alive with two successive negative SARS-Cov-2 qRT-PCR tests; (3) interval from symptoms to hospital admission, discharge or death; (4) negative qRT-PCT test; and (5) interval from admission to COVID-19 progression. Data collection forms were reviewed and verified independently by two physicians (WH and JY). A third researcher adjudicated discordances. Data were analyzed as of 24 April 2020.

\section{Definitions}

Solid cancers and hematological cancers were included. Cancer remission was defined as complete remission for $\geq 1$ year. Exposure history was defined as contact with persons with confirmed SARS-CoV-2 infection or visit to the South China Seafood Wholesale Market. Smoking history was defined as current or former smoker (stopping $>5$ years ago) with exposure of $\geq 20$ cigarettes per day for $\geq 1$ year ( 1 pack year). Fever was defined as temperature $\geq 37.3{ }^{\circ} \mathrm{C}$. Acute kidney and heart injury and acute respiratory distress syndrome (ARDS) were defined as reported [32, 33]. Liver injury was defined as $\geq 1$ liver function enzyme activity value $\geq 2 \mathrm{x}$ upper limit of normal. Invasive and non-invasive mechanical ventilations were defined as mechanical ventilation with or without intubation $[34,35]$.

\section{Statistical analysis}

Continuous variables with a skewed distribution were described as median (interquartile range, IQR). Categorical variables are presented as number (\%). Proportions for categorical variables were compared by $\chi 2$ test or Fisher exact test. Pearson $\chi^{2}$ test was used for contingency data for variables with more than two categories. Mann-Whitney test was used to compare continuous variables. Co-variates associated with risk of in-hospital death from COVID-19 were analyzed in a Cox regression model. Hazard ratios (HR) and corresponding 95\% confidence intervals (CI) calculated using the Cox proportional hazard model. Covariates with $P<0.05$ in uni-variable analyses except those with co-linearity were included in multi-variable analyses. Statistical analysis was performed with SPSS 26.0 statistical software (IBM Corporation, New York, United States). A two-sided $P$ value $<0.05$ was considered significant.

\section{Results}

\section{Admission demographic and baseline co-variates}

From 20 January to 4 April 2020, 3559 hospitalized persons who died or were discharged with COVID-19 were enrolled, 1859 of whom had quantitative real-time reverse transcriptase-polymerase chain reaction (qRT-PCR)- $(N=$ $1790)$ or IgM/G $(N=69)$-confirmed SARS-CoV-2-infection were included in the analysis. 1650 survived; 209 died. Age and age distribution, sex, smoking history, exposure history, co-morbidities, signs and symptoms at admission, radiological features by lung computed tomography (CT) scan and COVID-19 severity were displayed in Table 1.

Of the 1859 subjects, 65 (3\%) had cancer 47 survived; 18 died. None of the 18 subjects died from cancer. Detailed data were available in 59 subjects (Supplement Table 1). Most had solid cancers including breast $(N=8)$, thyroid $(N=7)$ and gastric cancers $(N=6) .9$ subjects had hematological cancers including lymphoma $(N=4)$, leukemia $(N=3)$, myelodysplastic syndrome $(N=1)$ and plasma cell myeloma $(N=1)$. Twenty-one subjects were diagnosed $>5$ years, $25,1-5$ years ago and $13, \leq 1$ year before their COVID-19 admission. In 41 subjects the cancer was in complete remission and in 18 in less than complete 
Table 1 Demographic and clinical co-variates.

\begin{tabular}{|c|c|c|c|c|}
\hline & $\begin{array}{l}\text { Total } \\
N=1859\end{array}$ & $\begin{array}{l}\text { Non-cancer } \\
N=1794 \text { (97) }\end{array}$ & $\begin{array}{l}\text { Cancer } \\
N=65 \text { (3) }\end{array}$ & $P$-value \\
\hline \multicolumn{5}{|l|}{ Co-variates } \\
\hline $\begin{array}{l}\text { Age, median (IQR), } \\
\text { years }\end{array}$ & $59(45,68)$ & $59(45,68)$ & $63(54,70)$ & 0.054 \\
\hline Age & & & & 0.194 \\
\hline$<65$ years & $1199(65)$ & $1162(65)$ & 37 & \\
\hline$\geq 65$ years & $660(36)$ & $632(35)$ & 28 & \\
\hline Male & $934(50)$ & $903(50)$ & 31 & 0.706 \\
\hline Smoking history & $111(6)$ & $105(6)$ & 6 & 0.019 \\
\hline Former smoker & $66(4)$ & $60(3)$ & 6 & \\
\hline Current smoker & $45(2)$ & $45(3)$ & 0 & \\
\hline Exposure history & & & & 0.036 \\
\hline $\begin{array}{l}\text { Huanan Seafood } \\
\text { Market }\end{array}$ & $4(0.2)$ & $3(0.2)$ & 1 & \\
\hline $\begin{array}{l}\text { Close contact with } \\
\text { infected persons }\end{array}$ & $78(4)$ & 77 (4) & 1 & \\
\hline \multicolumn{5}{|l|}{ Co-morbidity } \\
\hline ASCVD & $268(14)$ & 259 (14) & 9 & $>0.999$ \\
\hline Arterial hypertension & $579(31)$ & $559(31)$ & 20 & $>0.999$ \\
\hline Diabetes mellitus & $262(14)$ & $250(14)$ & 12 & 0.303 \\
\hline COPD & $61(3)$ & $60(3)$ & 1 & 0.722 \\
\hline $\begin{array}{l}\text { Chronic kidney } \\
\text { disease }\end{array}$ & $45(2)$ & $44(3)$ & 1 & $>0.999$ \\
\hline $\begin{array}{l}\text { Gastro-intestinal } \\
\text { disease }\end{array}$ & $98(5)$ & $93(5)$ & 5 & 0.388 \\
\hline \multicolumn{5}{|l|}{ Signs and symptoms } \\
\hline Fever $^{a}$ & $1448(78)$ & $1399(78)$ & 49 & 0.608 \\
\hline Temperature $\left({ }^{\circ} \mathrm{C}\right)^{\mathrm{b}}$ & $36.6(36.4,37.0)$ & $36.6(36.4,37.1)$ & $36.6(36.5,36.9)$ & 0.464 \\
\hline Shortness of breath & $716(39)$ & $685(38)$ & 31 & 0.123 \\
\hline Dry cough & $619(43)$ & $597(43)$ & 22 & 0.294 \\
\hline Wet cough & 715 (39) & $684(38)$ & 31 & 0.12 \\
\hline Fatigue & 695 (37) & $670(37)$ & 25 & 0.858 \\
\hline $\begin{array}{l}\text { Nausea and/or } \\
\text { vomiting }\end{array}$ & $124(9)$ & $116(8)$ & 8 & 0.21 \\
\hline Diarrhea & $243(13)$ & 234 (13) & 9 & 0.852 \\
\hline Chills & 281 (19) & $274(20)$ & 7 & 0.137 \\
\hline Rhinorrhea & $33(2)$ & $32(2)$ & 1 & $>0.999$ \\
\hline Myalgia & 315 (17) & 305 (17) & 10 & 0.867 \\
\hline Headache & $107(6)$ & $105(6)$ & 2 & 0.583 \\
\hline \multicolumn{5}{|l|}{ Radiological co-variates } \\
\hline Bilateral pneumonia & $1570(88)$ & $1515(88)$ & 55 & 0.693 \\
\hline Consolidation & $326(19)$ & $314(18)$ & 12 & 0.754 \\
\hline Ground-glass opacity & $1331(75)$ & $1287(75)$ & 44 & 0.6 \\
\hline Patchy shadows & $736(42)$ & $700(41)$ & 36 & 0.005 \\
\hline COVID-19 severity & & & & 0.003 \\
\hline Mild & $34(2)$ & $33(2)$ & 1 & \\
\hline Moderate & $1170(63)$ & $1141(64)$ & 29 & \\
\hline Severe & $453(24)$ & $433(24)$ & 20 & \\
\hline Critical & $202(11)$ & $187(10)$ & 15 & \\
\hline
\end{tabular}

Data are median (IQR) or $n(\%)$.

$A S C V D$ cardio- and cerebro-vascular disease, $C O P D$ chronic obstructive pulmonary disease

${ }^{\mathrm{a}} \geq 1$ temperature $\geq 37.3^{\circ} \mathrm{C}$ from onset of symptoms to admission.

${ }^{\mathrm{b}}$ Admission temperature.

remission. No subjects were receiving anti-cancer therapy on admission for COVID-19. Sixteen of them received anticancer therapy $<1$ month before admission. Therapies included surgery $(N=1)$, chemotherapy $(N=12)$, targeted therapy $(N=6)$ and radiation therapy $(N=1)$. Demographic and clinical characteristics of COVID-19 subjects with cancers were shown in Supplement Table 2.
Comparison of cancer and non-cancer subjects with COVID-19 by admission clinical co-variates

Subjects with cancer were more likely to be old (median 63 years, interquartile range [IQR] $54-70$ years versus 59 years, [IQR] 46-68 years; $P=0.054$; Table 1 ), to be smokers $(9 \%$ versus $6 \%, P=0.019)$ and to have visited the Huanan Seafood Whole Sale Market (1 versus 3; $P=$ 0.036). There were no significant differences in age distribution (57\% versus $65 \%,<65$ years; $P=0.194$ ) or $\geq 1$ prior co-morbidity ( $46 \%$ versus $47 \%$; $P>0.999$ ). Prior comorbidities included ASCVD $(N=9)$, arterial hypertension $(N=20)$, diabetes mellitus $(N=12)$, COPD $(N=1)$, chronic kidney disease $(N=1)$ and gastro-intestinal disease $(N=5)$.

Signs and symptoms on admission including temperature, shortness of breath, dry and wet coughs, fatigue, nausea and/or vomiting, diarrhea, chills, rhinorrhea, myalgia and headache were similar between subjects with and without cancer. Subjects with cancer subjects were more likely to have patchy lung infiltrates on lung CT (60\% versus $41 \%, P=0.005$ ) but similar frequencies of consolidation, bilateral pneumonia and ground-glass opacity. Subjects with cancer were more likely to have severe/critical COVID-19 severity on admission (54\% versus 35\%; $P=0.003)$.

\section{Comparison of cancer and non-cancer subjects with COVID-19 by laboratory co-variates}

Admission laboratory co-variates in subjects with and without cancer are displayed in Table 2. Cancer subjects had significant higher median concentrations of neutrophils $(5 \times 10 \mathrm{E}+9 / \mathrm{L}[\mathrm{IQR} 3-7 \times 10 \mathrm{E}+9 / \mathrm{L}]$ versus $3 \times 10 \mathrm{E}+$ 9/L [IQR $2-5 \times 10 \mathrm{E}+9 / \mathrm{L}] ; P=0.001)$, neutrophil-tolymphocyte ratio (NLR) (4 [IQR 3-8] versus 3 [IQR 2-5]; $P<0.001)$ and lower median concentrations of lymphocytes $(0.9 \times 10 \mathrm{E}+9 / \mathrm{L}[\mathrm{IQR} 0.6-1.3 \times 10 \mathrm{E}+9 / \mathrm{L}]$ versus $1.1 \times$ $10 \mathrm{E}+9 / \mathrm{L}[\mathrm{IQR} 0.8-1.6 \times 10 \mathrm{E}+9 / \mathrm{L}] ; P<0.001)$ and hemoglobin $(120 \times \mathrm{g} / \mathrm{L}$ [IQR $102-132 \times \mathrm{g} / \mathrm{L}]$ versus $128 \times$ $\mathrm{g} / \mathrm{L}[\mathrm{IQR} 117-139 \times \mathrm{g} / \mathrm{L}] ; P<0.001)$. There were no significant differences in platelet concentrations or percentages of lymphocyte subsets including CD3-positive cells, CD4positive cells, CD8-positive cells, natural-killer (NK) cells or B lymphocytes or in CD4-positive/CD8-positive ratio. Median plasma concentration of tumor necrosis factor (TNF)- $\alpha$ was lower in subjects with cancer $(2.9 \mathrm{pg} / \mathrm{ml}$ $[1.8-3.5 \mathrm{pg} / \mathrm{ml}]$ versus $3.4 \mathrm{pg} / \mathrm{ml} \quad[2.2-5.5 \mathrm{pg} / \mathrm{ml}] ; P=$ 0.029). Plasma concentrations of other cytokines including interleukin (IL)-4, IL-6, IL-10, and interferon (IFN)- $\gamma$ were similar.

Cancer subjects had higher median serum concentrations of C-reactive protein (CRP, $27 \mathrm{mg} / \mathrm{L}$ [IQR $6-66 \mathrm{mg} / \mathrm{L}$ ] 
Table 2 Laboratory co-variates.

\begin{tabular}{|c|c|c|c|c|c|}
\hline Co-variates (normal range) & $N$ & Total & Non-cancer & Cancer & $P$ value \\
\hline \multicolumn{6}{|l|}{$\mathrm{CBC}$} \\
\hline Neutrophils $\times 10 \mathrm{E}+9 / \mathrm{L}(1.8-6.3)$ & 1816 & $3(2,5)$ & $3(2,5)$ & $5(3,7)$ & 0.001 \\
\hline Lymphocytes ×10 E + 9/L (1.1-3.2) & 1847 & $1.0(0.8,1.6)$ & $1.1(0.8,1.6)$ & $0.9(0.6,1.3)$ & 0.001 \\
\hline Monocytes ×10 E + 9/L (0.1-0.6) & 1805 & $0.4(0.3,0.5)$ & $0.4(0.3,0.5)$ & $0.4(0.2,0.7)$ & 0.32 \\
\hline Hemoglobin, g/L (115-150) & 1433 & $128(117,139)$ & $128(117,139)$ & $120(102,132)$ & $<0.001$ \\
\hline Platelets $\times 10 \mathrm{E}+9 / \mathrm{L}(125-350)$ & 1814 & $203(155,264)$ & $203(155,263)$ & $216(157,284)$ & 0.479 \\
\hline NLR & 1814 & $3(2,5)$ & $3(2,5)$ & $4(3,8)$ & $<0.001$ \\
\hline \multicolumn{6}{|l|}{ Inflammation co-variates } \\
\hline $\mathrm{CRP}, \mathrm{mg} / \mathrm{L}(<8)$ & 1371 & $13(3,51)$ & $13(3,49)$ & $27(6,66)$ & 0.047 \\
\hline Procalcitonin, $\mathrm{ng} / \mathrm{ml}(<0.5)$ & 1643 & $0.06(0.05,0.1)$ & $0.05(0.05,0.1)$ & $0.1(0.06,0.2)$ & 0.001 \\
\hline LDH, U/L (109-245) & 1729 & $212(170,292)$ & $211(170,289)$ & $252(196,391)$ & $<0.001$ \\
\hline Ferritin, ng/ml (4.6-204) & 308 & $567(246,1218)$ & $553(242,1209)$ & $817(323,1599)$ & 0.119 \\
\hline \multicolumn{6}{|l|}{ Coagulation co-variates } \\
\hline aPTT, s (28-43.5) & 1356 & $34(30,38)$ & $34(30,38)$ & $35(32,40)$ & 0.009 \\
\hline Fibrinogen, g/L (2-4) & 1323 & $3.7(2.9,4.6)$ & $3.7(2.9,4.6)$ & $3.8(3.4,4.6)$ & 0.222 \\
\hline D-dimer, mg/L $(<0.5)$ & 1602 & $0.4(0.2,1.1)$ & $0.4(0.2,1.0)$ & $0.6(0.2,4.0)$ & 0.043 \\
\hline \multicolumn{6}{|l|}{ Biochemistry co-variates } \\
\hline ALT, U/L (5-35) & 1832 & $38(22,67)$ & $38(22,67)$ & $36(24,67)$ & 0.882 \\
\hline AST, U/L (8-40) & 1830 & $32(22,49)$ & $32(22,49)$ & $35(27,58)$ & 0.039 \\
\hline Total bilirubin, $\mu \mathrm{mol} / \mathrm{L}(5.1-19)$ & 1586 & $14(10,19)$ & $14(10,19)$ & $15(11,29)$ & 0.069 \\
\hline Creatine kinase, U/L (26-140) & 1493 & $88(54,165)$ & $88(54,163)$ & $102(52,282)$ & 0.164 \\
\hline BNP, pg/ml $(<100)$ & 838 & $61(18,242)$ & $60(17,238)$ & $85(30,679)$ & 0.042 \\
\hline Myoglobin, ng/ml (<140) & 972 & $35(21,73)$ & $34(21,71)$ & $41(31,303)$ & 0.004 \\
\hline Troponin I, ng/L (<26.2) & 1083 & $4(1,14)$ & $4(1,14)$ & $8(3,34)$ & 0.006 \\
\hline BUN, mmol/L (2.9-8.2) & 1815 & $4.9(3.9,6.7)$ & $4.9(3.9,6.7)$ & $5.3(3.8,9.9)$ & 0.255 \\
\hline Scr, $\mu \mathrm{mol} / \mathrm{L}(44-106)$ & 1813 & $71(59,85)$ & $71(60,85)$ & $65(55,82)$ & 0.076 \\
\hline \multicolumn{6}{|l|}{ Lymphocyte subsets } \\
\hline $\mathrm{CD} 3+,(58-84 \%)$ & 759 & $71(62,78)$ & $72(62,78)$ & $68(58,82)$ & 0.877 \\
\hline $\mathrm{CD} 4+,(25-51 \%)$ & 759 & $41(32,48)$ & $41(33,48)$ & $39(29,49)$ & 0.373 \\
\hline $\mathrm{CD} 8+,(14-39 \%)$ & 759 & $23(17,30)$ & $23(17,30)$ & $21(17,32)$ & 0.793 \\
\hline NK-cells $(3-30 \%)$ & 561 & $10(6,16)$ & $10(6,16)$ & $8(5,18)$ & 0.688 \\
\hline B-cells $(4-18 \%)$ & 561 & $12.6(8.6,18)$ & $12.7(8.7,18)$ & $12.6(6.2,19.3)$ & 0.828 \\
\hline $\mathrm{CD} 4+/ \mathrm{CD} 8+$ ratio $(0.41-2.72)$ & 755 & $1.9(1.4,2.7)$ & $1.9(1.4,2.7)$ & $2.1(1.4,3.1)$ & 0.462 \\
\hline \multicolumn{6}{|l|}{ Cytokines } \\
\hline $\mathrm{IL}-4, \mathrm{pg} / \mathrm{ml}(0.1-3.2)$ & 505 & $3(2,4)$ & $3(2,4)$ & $2(1,3)$ & 0.32 \\
\hline IL-6, pg/ml (0.1-2.9) & 857 & $10(4,42)$ & $10(4,42)$ & $17(7,102)$ & 0.085 \\
\hline $\mathrm{IL}-10, \mathrm{pg} / \mathrm{ml}(0.1-5)$ & 505 & $4(3,6)$ & $4.3(2.9,5.6)$ & $4.4(2.7,7.9)$ & 0.642 \\
\hline TNF- $\alpha, p g / m l(0.1-23)$ & 505 & $3.3(2.2,5.4)$ & $3.4(2.2,5.5)$ & $2.9(1.8,3.5)$ & 0.029 \\
\hline IFN- $\gamma, \mathrm{pg} / \mathrm{ml}(0.1-18)$ & 505 & $3.1(2.0,4.1)$ & $3.1(2.0,4.1)$ & $2.7(1.8,4.0)$ & 0.447 \\
\hline
\end{tabular}

Data are median (IQR).

$N L R$ neutrophil-to-lymphocyte ratio, $C R P$ c-reactive protein, $L D H$ lactate dehydrogenase, $a P T T$ activated partial thromboplastin time, $A L T$ alanine aminotransferase, $A S T$ aspartate aminotransferase, $B N P$ B-type natriuretic peptide, $B U N$ blood urea nitrogen, $S c r$ serum creatinine, $I L$ interleukin, $T N F$ tumor necrosis factor, IFN interferon.

versus $13 \mathrm{mg} / \mathrm{L}$ [IQR $3-49 \mathrm{mg} / \mathrm{L}] ; P=0.047)$, procalcitonin $(0.1 \mathrm{ng} / \mathrm{ml}[\mathrm{IQR} 0.06-0.2 \mathrm{ng} / \mathrm{ml}]$ versus $0.05 \mathrm{ng} / \mathrm{ml}[\mathrm{IQR}$ $0.05-0.1 \mathrm{ng} / \mathrm{ml}] ; \quad P=0.001)$, and D-dimer $(0.6 \mathrm{mg} / \mathrm{L}$ [IQR $0.2-4.0 \mathrm{mg} / \mathrm{L}$ ] versus $0.4 \mathrm{mg} / \mathrm{L}$ [IQR $0.2-1.0 \mathrm{mg} / \mathrm{L}$ ];
$P=0.043)$ and longer activated partial thromboplastin time (aPTT, median $35 \mathrm{~s}$ [IQR 32-40 s] versus $34 \mathrm{~s}$ [IQR 30-38 s]; $P=0.009$ ). No significant difference was found in serum concentrations of ferritin or fibrinogen. 
Cancer subjects had higher median activities of lactate dehydrogenase (LDH) (252 U/L [IQR 196-391 U/L] versus 211 U/L [IQR 170-289 U/L]; $P<0.001$ ), aspartate aminotransferase (AST, 35 U/L [27-58 U/L] versus $32 \mathrm{U} / \mathrm{L}$ [22-49 U/L]; $\quad P=0.039), \quad$ concentrations of B-type natriuretic peptide (BNP, $85 \mathrm{pg} / \mathrm{ml}[30-679 \mathrm{pg} / \mathrm{ml}]$ versus $60 \mathrm{pg} / \mathrm{ml}[17-238 \mathrm{pg} / \mathrm{ml}] ; P=0.042)$, myoglobin $(41 \mathrm{ng} / \mathrm{ml}$ [31-303 ng/ml] versus $34 \mathrm{ng} / \mathrm{ml}[21-71 \mathrm{ng} / \mathrm{ml}] ; P=0.004)$ and troponin I ( $8 \mathrm{ng} / \mathrm{L}[3-34 \mathrm{ng} / \mathrm{L}]$ versus $4 \mathrm{ng} / \mathrm{L}$ [1-14 ng/ $\mathrm{L}] ; P=0.006)$. There were no significant differences in activities of alanine aminotransferase (ALT) or creatine kinase or concentrations of total bilirubin, blood urea nitrogen (BUN) or serum creatinine (Scr).

\section{Complications and outcomes of subjects with cancer and without cancer}

As shown in Table 3, subjects with cancer had higher incidence of ARDS (19 [29\%] versus 208 [12\%]; $P<0.001)$, bacterial infection (30 [46\%] versus $533[30 \%] ; P=0.005)$,

Table 3 Complications and therapy.

\begin{tabular}{|c|c|c|c|c|}
\hline & $\begin{array}{l}\text { Total } \\
N=1859\end{array}$ & $\begin{array}{l}\text { Non-cancer } \\
N=1794\end{array}$ & $\begin{array}{l}\text { Cancer } \\
N=65\end{array}$ & $P$-value \\
\hline \multicolumn{5}{|l|}{ Complications } \\
\hline ARDS & $227(12)$ & $208(12)$ & 19 & $<0.001$ \\
\hline Bacterial infections & $563(30)$ & $533(30)$ & 30 & 0.005 \\
\hline Septic shock & $64(4)$ & $57(4)$ & 7 & 0.007 \\
\hline Acute kidney injury & $99(5)$ & $90(5)$ & 9 & 0.002 \\
\hline Heart injury & $229(12)$ & $215(12)$ & 14 & 0.021 \\
\hline Abnormal LFT & $456(25)$ & $440(25)$ & 16 & 0.984 \\
\hline Gastro-intestinal bleeding & $34(2)$ & $29(2)$ & 5 & $<0.001$ \\
\hline Coagulopathy & $49(3)$ & $47(3)$ & 2 & 0.821 \\
\hline Multiple organ failure & $129(7)$ & $117(7)$ & 12 & $<0.001$ \\
\hline \multicolumn{5}{|l|}{ Therapy } \\
\hline Antibiotics & $1559(85)$ & $1497(85)$ & 62 & 0.019 \\
\hline Anti-fungal drugs & $71(4)$ & $64(4)$ & 7 & 0.005 \\
\hline Oseltamivir & $757(41)$ & $729(41)$ & 28 & 0.697 \\
\hline Umifenovir & $1386(75)$ & $1343(75)$ & 43 & 0.110 \\
\hline Lopinavir/ritonavir & $339(23)$ & $328(24)$ & 11 & 0.357 \\
\hline Interferon alpha & $387(21)$ & $363(20)$ & 24 & 0.001 \\
\hline Corticosteroids & $753(41)$ & $725(40)$ & 28 & 0.670 \\
\hline IVIG & $506(29)$ & $478(29)$ & 28 & 0.009 \\
\hline High-flow nasal cannula oxygen therapy & $233(16)$ & $214(15)$ & 19 & 0.001 \\
\hline Non-invasive mechanical ventilation & $145(8)$ & $130(7)$ & 15 & $<0.001$ \\
\hline Invasive mechanical ventilation & $85(5)$ & $78(4)$ & 7 & 0.015 \\
\hline ECMO & $4(0.2)$ & $4(0.2)$ & 0 & $>0.999$ \\
\hline CRRT & $23(1)$ & $22(1)$ & 1 & 0.561 \\
\hline \multicolumn{5}{|l|}{ Outcomes } \\
\hline Death & $209(11)$ & $191(11)$ & 18 & $<0.001$ \\
\hline Time from illness onset to ICU admission, median (IQR), days & $14(10,20)$ & $14(10,20)$ & $14(7,21)$ & 0.790 \\
\hline Time from illness onset to repeated negative SARS-CoV-2 tests, median (IQR), days & $22(17,28)$ & $22(17,28)$ & $20(16,28)$ & 0.379 \\
\hline Time from illness onset to admission, median (IQR), days & $10(7,15)$ & $10(7,15)$ & $10(7,13)$ & 0.104 \\
\hline Time from illness onset to progression, median (IQR), days & $10(7,15)$ & $10(5,14)$ & $11(6,18)$ & 0.284 \\
\hline Time from illness onset to outcome ${ }^{\mathrm{a}}$, median (IQR), days & $30(23,37)$ & $30(23,37)$ & $28(21,35)$ & 0.209 \\
\hline Time from diagnosis to outcome ${ }^{\mathrm{a}}$, median (IQR), days & $19(13,27)$ & $19(13,27)$ & $21(14,27)$ & 0.503 \\
\hline Time from admission to outcome ${ }^{\mathrm{a}}$, median (IQR), days & $18(12,23)$ & $18(12,23)$ & $18(13,26)$ & 0.353 \\
\hline
\end{tabular}

LFT liver function test, ARDS acute respiratory distress syndrome, IVIG intravenous immunoglobin, ECMO extra-corporeal membrane oxygenation, $C R R T$ continuous renal replacement therapy, ICU intensive care unit.

${ }^{a}$ Outcome refers to discharged alive or died. 
septic shock (7 [11\%] versus 57 [4\%], $P=0.007)$, acute kidney injury (9 [14\%] versus $90[5 \%] ; P=0.002)$, cardiac injury (14 [22\%] versus $215[12 \%] ; P=0.021)$, gastrointestinal bleeding $(5[8 \%]$ versus $29[2 \%] ; P<0.001)$ and multiple organ failure (12 [19\%] versus 117 [7\%]; $P<0.001)$.

Subjects with cancer were also more likely to receive antibiotics (62 [95\%] versus 1497 [85\%]; $P=0.019)$, antifungal drugs (7 [11\%] versus $64[4 \%] ; P=0.005)$, intratracheal $\alpha$-interferon (24 [37\%] versus 363 [20\%]; $P=$ 0.001 ), immunoglobin intravenous (IVIG, 28 [44\%] versus 478 [29\%]; $P=0$.009), high-flow nasal cannula oxygen (19 [31\%] versus 214 [15\%]; $P=0.001)$, invasive (7 [11\%] versus 78 [4\%]; $P=0.015)$ and non-invasive (15 [23\%] versus 130 [7\%]; $P<0.001)$ mechanical ventilation. There was no significant difference in use of extra-corporeal membrane oxygenation (ECMO) or continuous renal replacement therapy (CRRT).

Subjects with cancer were more likely to die from COVID-19 (18 [28\%] versus 191 [11\%]; $P<0.001)$. There were no significant differences in interval from hospital admission to ICU admission, repeatedly negative SARSCoV-2 tests, interval from admission to recovery, COVID19 progression or death.

\section{Multi-variable analyses}

We analyzed co-variates associated with risk of in-hospital death from COVID-19 in a Cox regression model including co-variates with $P<0.05$ in uni-variable analyses excluding those with co-linearity (Supplement Table 2). Age per year increase $(\mathrm{HR}=1.05$ [1.04, 1.07]; $P<0.001)$, male $(\mathrm{HR}=$ $1.53[1.09,2.14] ; \quad P=0.013)$, admission COVID-19 severity severe/critical $\quad(\mathrm{HR}=28.2 \quad[13.8,57.6] ; \quad P<$ 0.001 ), current smoking (HR $2.00[1.10,3.66] ; P=0.024)$, admission temperature $\mathrm{per}{ }^{\circ} \mathrm{C}(\mathrm{HR}=1.24[1.03,1.50] ; P=$ 0.026), platelet concentration per $10 \mathrm{E}+9 / \mathrm{L}$ increase $(\mathrm{HR}=0.996$ [0.994, 0.998]; $P=0.001)$ and plasma Ddimer concentration $p e r \mathrm{mg} / \mathrm{L}$ increase $(\mathrm{HR}=1.04$ [1.02, 1.05]; $P<0.001)$ but not having cancer $(\mathrm{HR}=1.59[0.95$,
2.69]; $P=0.081$; Table 4) were significantly associated with risk of in-hospital death from COVID-19.

Next, we performed separate multi-variable regression analyses in subjects $<$ and $\geq 65$ years after adjusting age and sex. We found having cancer was significantly associated with risk of in-hospital death from COVID-19 in subjects $<65$ years $(\mathrm{HR}=2.45[1.04,5.76] ; P=0.041)$ but not in subjects $\geq 65$ years $(\mathrm{HR}=1.12[0.56,2.24]$; $P=0.740)$. Other risk factors in subjects $<65$ years included age per year increase (HR $=1.05$ [1.02, 1.09]; $P=0.001$, COVID19 severity severe/critical at admission $(\mathrm{HR}=16.1 \quad[6.3$, 40.8]; $P<0.001)$ and plasma D-dimer concentration per $\mathrm{mg} / \mathrm{L}$ increase $(\mathrm{HR}=1.21 \quad[1.08,1.17] ; P<0.001)$. In subjects $\geq 65$ years age per year increase (HR 1.05 [1.02, 1.07]; $P<0.001)$, admission COVID-19 severity severe/ critical $(\mathrm{HR}=46.1$ [14.6, 145.9]; $P<0.001)$, platelet concentration per $10 \mathrm{E}+9 / \mathrm{L}$ increase $(\mathrm{HR}=0.996$ [0.9930.998]; $P=0.002$ ) and plasma D-dimer concentration per $\mathrm{mg} / \mathrm{L}$ increase (HR 1.03 [1.01, 1.04]; $P<0.001$ ) were correlated with risk of in-hospital death from COVID-19.

\section{Comparison of co-variates in subjects with cancer living or dying from COVID-19}

18 subjects with cancer died. There was no difference in median age (68 years, IQR 56-78 years versus 62 years, IQR 51-67 years; $P=0.146$, Supplement Table 3), sex $(P=0.58)$ or frequencies of prior co-morbidities between non-survivors and survivors. Non-survivors were more likely to have had shortness of breath on admission $(78 \%$ versus $36 \% ; P=0.005$ ) but there were no significant differences in other signs or symptoms on admission or in findings on lung CT scan. Non-survivors were more likely to have had severe/ critical COVID-19 on admission than survivors (94\% versus $38 \%, P<0.001)$. Non-survivors had significantly higher median concentrations/activities of neutrophils, CRP, procalcitonin, LDH, ferritin, D-dimer, ALT, AST, total bilirubin, creatine kinase, BNP, myoglobin, troponin I, BUN, Scr, and NLR, and lower

Table 4 Multi-variable analyses of co-variates associated with in-hospital death of persons with cancer.

\begin{tabular}{|c|c|c|c|c|c|c|}
\hline & \multicolumn{2}{|l|}{ All } & \multicolumn{2}{|l|}{$<65$ years } & \multicolumn{2}{|l|}{$\geq 65$ years } \\
\hline & HR $(95 \% \mathrm{CI})$ & $P$ value & HR $(95 \% \mathrm{CI})$ & $P$ value & HR $(95 \%$ CI $)$ & $P$ value \\
\hline Age, years & $1.05(1.04,1.07)$ & $<0.001$ & $1.05(1.02,1.09)$ & 0.001 & $1.05(1.02,1.07)$ & $<0.001$ \\
\hline Male & $1.53(1.09,2.14)$ & 0.013 & $1.82(0.999,3.30)$ & 0.050 & $1.50(0.998,2.27)$ & 0.051 \\
\hline Severity (severe/critical) & $28.17(13.78,57.62)$ & $<0.001$ & $16.06(6.32,40.80)$ & $<0.001$ & $46.10(14.56,145.91)$ & $<0.001$ \\
\hline Cancer & $1.59(0.95,2.69)$ & 0.081 & $2.45(1.04,5.76)$ & 0.041 & $1.12(0.56,2.24)$ & 0.740 \\
\hline Current smoking & $2.00(1.10,3.66)$ & 0.024 & $1.22(0.37,4.02)$ & 0.741 & $2.43(1.20,4.91)$ & 0.014 \\
\hline Temperature at admission $\left({ }^{\circ} \mathrm{C}\right)$ & $1.24(1.03,1.50)$ & 0.026 & $1.09(0.79,1.50)$ & 0.609 & $1.35(1.05,1.73)$ & 0.021 \\
\hline Platelets $\times 10 E+9 / L$ & $0.996(0.994,0.998)$ & 0.001 & $0.997(0.994,1.001)$ & 0.099 & $0.996(0.993,0.998)$ & 0.002 \\
\hline D-dimer, mg/L & $1.04(1.02,1.05)$ & $<0.001$ & $1.21(1.08,1.17)$ & $<0.001$ & $1.03(1.01,1.04)$ & $<0.001$ \\
\hline
\end{tabular}


concentrations of lymphocytes (Supplement Table 4). Percentages of CD3-, CD4- and CD8-positive T-cells, CD4/ CD8 ratio and B-cells were similar between survivors and non-survivors. In contrast, percentage of natural killer (NK)-cells was significantly lower in non-survivors (4\% [IQR 2-6\%] versus $14 \%$ [IQR 7-18\%]; $P=0.008)$. Nonsurvivors had higher concentrations of IL-6 $(287 \mathrm{pg} / \mathrm{ml}$ [IQR $22-686 \mathrm{pg} / \mathrm{ml}$ ] versus $12 \mathrm{pg} / \mathrm{ml}$ [IQR $5-27 \mathrm{pg} / \mathrm{ml}$; $P$ $=0.001)$ and IL-10 $(10 \mathrm{pg} / \mathrm{ml}[\mathrm{IQR} 4-18 \mathrm{pg} / \mathrm{ml}]$ versus 4 $\mathrm{pg} / \mathrm{ml} \quad[\mathrm{IQR} \quad 3-5 \mathrm{pg} / \mathrm{ml}] ; P=0.012$ ). There was no significant difference in serum concentrations of IL- 4 , TNF- $\alpha$ or IFN- $\gamma$. Time from admission to recovery, discharge or death was significantly shorter in non-survivors (21 days [IQR 15-30 days] versus 31 days [IQR 24-37 days]; $P=$ 0.002; Supplement Table 5).

We further compared co-variates between subjects with solid and hematological cancers (Supplement Table 6). Subjects with hematological cancer were more likely to be young (39 years [IQR 23-66 years] versus 63 years [IQR 57-69 years]; $P=0.019$ ), to have lower hemoglobin concentration $(92 \mathrm{~g} / \mathrm{L}$ [IQR $86-116 \mathrm{~g} / \mathrm{L}$ ] versus $121 \mathrm{~g} / \mathrm{L}$ [IQR $106-133 \mathrm{~g} / \mathrm{L}] ; P=0.011)$ and longer aPTT (42 s [IQR $38-48 \mathrm{~s}$ ] versus $35 \mathrm{~s}$ [IQR $32-38 \mathrm{~s}] ; P=0.001)$. There was no difference in other co-variates including signs and symptoms, laboratory co-variates or co-morbidities on admission. Rates of ICU admission bacterial co-infections, of ARDS and of in-hospital death from COVID-19 (2/9 versus $14 / 50 ; P>0.999)$ were similar. We also compared co-variates in subjects in complete remission or not. (Supplement Table 7) Subjects not in complete remission were more likely to be young (54 years [IQR 27-66 years] versus 63 years [IQR 58-71 years]; $P=0.034$ ), to have a lower incidence of diabetes and lower median concentrations of hemoglobin $(100 \mathrm{~g} / \mathrm{L}$ [IQR $89-107 \mathrm{~g} / \mathrm{L}]$ versus $129 \mathrm{~g} / \mathrm{L}$ [IQR $118-137 \mathrm{~g} / \mathrm{L}] ; P<0.001)$ and plasma IL-6 $(43 \mathrm{pg} / \mathrm{ml}$ $[13-593 \mathrm{pg} / \mathrm{ml}]$ versus $11 \mathrm{pg} / \mathrm{ml}[3-37 \mathrm{pg} / \mathrm{ml}] ; P=0.022)$ and a higher risk of death $(9 / 18$ versus $7 / 41 ; P=0.013)$.

\section{Discussion}

There is controversy whether having cancer is associated with an increased risk of death from COVID-19. Some studies report an association [1-3, 5-9, 13-18], others not [19-26]. These differences could be related to cancer patients' age or other factors. We studied 1859 consecutive subjects with COVID-19, 65 of whom also had cancer. Detailed data were available in 59. The strongest risk factor for in-hospital death from COVID-19 in all subjects and in subjects with cancer was COVID-19 severity on admission. However, we also found having cancer increased risk of inhospital death from COVID-19 in subjects $<65$ years but not in those $\geq 65$ years. We also found risk of death in persons with cancer only operated in those $<65$ years and in those not in complete remission. Type of cancer (solid or hematological) was not significantly correlated with risk of in-hospital death from COVID-19.

There are limitations to our study. It was retrospective, has relatively few subjects with cancer but $>1800$ controls and lacks a validation cohort. Nevertheless, we think our data may be informative in treating persons with cancer and COVID-19.

\section{Data availability}

All data are included in the typescript and supplement.

Acknowledgements We thank participating patients, families and health care providers. Supported by the National Natural Science Foundation of China (81974009 to QL and 81974221 to ZC) and the Fundamental Research Funds for the Central Universities (2020kfyXGYJ086 to QL). RPG acknowledges support from the National Institute of Health Research (NIHR) Biomedical Research Centre funding scheme.

Author contributions YH and QL designed the study. Lei Chen, Q Li, WH, JY, Li Chen, YC, WC, DW, FD, LC, QR, LL, QL, WR, FG, $\mathrm{HW}$, and ZC collected the data. All authors had full access to the data, were involved in data interpretation and vouch for the accuracy of the analyses. QL, Lei Chen, and RPG prepared the typescript which all authors approved and agreed to submit for publication.

\section{Compliance with ethical standards}

Conflict of interest The authors declare that they have no conflict of interest.

Publisher's note Springer Nature remains neutral with regard to jurisdictional claims in published maps and institutional affiliations.

\section{References}

1. Liang W, Guan W, Chen R, Wang W, Li J, Xu K, et al. Cancer patients in SARS-CoV-2 infection: a nationwide analysis in China. Lancet Oncol. 2020;21:335-7.

2. He W, Chen L, Chen L, Yuan G, Fang Y, Chen W, et al. COVID19 in persons with haematological cancers. Leukemia. 2020;34:1637-45.

3. Tang LV, Hu Y Poor clinical outcomes for patients with cancer during the COVID-19 pandemic. Lancet Oncol. 2020. https://doi. org/10.1016/S1470-2045(20)30311-9.

4. Li H, Chen C, Hu F, Wang J, Zhao Q, Gale RP, et al. Impact of corticosteroid therapy on outcomes of persons with SARS-CoV-2, SARS-CoV, or MERS-CoV infection: a systematic review and meta-analysis. Leukemia 2020;34:1503-11.

5. Dai M, Liu D, Liu M, Zhou F, Li G, Chen Z, et al. Patients with cancer appear more vulnerable to SARS-COV-2: a multi-center study during the COVID-19 outbreak. Cancer Discov. 2020. https://doi.org/10.1158/2159-8290.CD-20-0422.

6. Yang K, Sheng Y, Huang C, Jin Y, Xiong N, Jiang K, et al. Clinical characteristics, outcomes, and risk factors for mortality in patients with cancer and COVID-19 in Hubei, China: a multicentre, retrospective, cohort study. Lancet Oncol 2020;2045:1-10. 
7. Martín-Moro F, Marquet J, Piris M, Michael BM, Sáez AJ, Corona M, et al. Survival study of hospitalised patients with concurrent COVID-19 and haematological malignancies. $\mathrm{Br} \mathrm{J}$ Haematol. 2020. https://doi.org/10.1111/bjh.16801.

8. Ma J, Yin J, Qian Y, Wu Y. Clinical characteristics and prognosis in cancer patients with COVID-19: a single center's retrospective study. J Infect. 2020. https://doi.org/10.1016/j.jinf.2020.04.006.

9. Cook G, Ashcroft AJ, Pratt G, Popat R, Ramasamy K, Kaiser M, et al. Real-world assessment of the clinical impact of symptomatic infection with severe acute respiratory syndrome coronavirus (COVID-19 disease) in patients with Multiple Myeloma receiving systemic anti-cancer therapy. Br J Haematol. 2020. https://doi.org/ 10.1111/bjh. 16874

10. Fattizzo B, Giannotta JA, Sciumè M, Cattaneo D, Bucelli C, Fracchiolla NS, et al. Reply to "COVID-19 in persons with haematological cancers": a focus on myeloid neoplasms and risk factors for mortality. Leukemia. 2020. https://doi.org/10.1038/ s41375-020-0877-y.

11. Baumann T, Delgado J, Montserrat E. CLL and COVID-19 at the Hospital Clinic of Barcelona: an interim report. Leukemia. 2020. https://doi.org/10.1038/s41375-020-0870-5.

12. Gale RP. Perspective: SARS-CoV-2, COVID-19 and Haematologists. Acta Haematol. 2020. https://doi.org/10.1159/000508021.

13. Zhang L, Zhu F, Xie L, Wang C, Wang J, Chen R, et al. Clinical characteristics of COVID-19-infected cancer patients: a retrospective case study in three hospitals within Wuhan, China. Ann Oncol. 2020. https://doi.org/10.1016/j.annonc.2020.03.296.

14. Mehta V, Goel S, Kabarriti R, Cole D, Goldfinger M, AcunaVillaorduna A, et al. Case fatality rate of cancer patients with COVID-19 in a New York hospital system. Cancer Discov. 2020. https://doi.org/10.1158/2159-8290.CD-20-0516.

15. Liang W, Liang H, Ou L, Chen B, Chen A, Li C, et al. Development and validation of a clinical risk score to predict the occurrence of critical illness in hospitalized patients with COVID19. JAMA Intern Med. 2020. https://doi.org/10.1001/jama internmed.2020.2033.

16. Guan W, Liang W, Zhao Y, Liang $\mathrm{H}$, Chen Z, Li Y, et al. Comorbidity and its impact on 1590 patients with COVID-19 in China: a nationwide analysis. Eur Respir J. 2020. https://doi.org/ 10.1183/13993003.00547-2020.

17. Tian J, Yuan X, Xiao J, Zhong Q, Yang C, Liu B, et al. Clinical characteristics and risk factors associated with COVID-19 disease severity in patients with cancer in Wuhan, China: a multicentre, retrospective, cohort study. Lancet Oncol. 2020. https://doi.org/ 10.1016/S1470-2045(20)30309-0

18. Kuderer NM, Choueiri TK, Shah DP, Shyr Y, Rubinstein SM, Rivera DR, et al. Clinical impact of COVID-19 on patients with cancer (CCC19): a cohort study. Lancet. 2020. https://doi.org/10. 1016/S0140-6736(20)31187-9.

19. Miyashita H, Mikami T, Chopra N, Yamada T, Chernyavsky S, Rizk D, et al. Do patients with cancer have a poorer prognosis of COVID-19? An experience in New York City. Ann Oncol. 2020. https://doi.org/10.1016/j.annonc.2020.04.006.

20. Petrilli CM, Jones SA, Yang J, Rajagopalan H, O'Donnell L, Chernyak Y, et al. Factors associated with hospital admission and critical illness among 5279 people with coronavirus disease 2019 in New York City: prospective cohort study. BMJ. 2020. https:// doi.org/10.1136/bmj.m1966.
21. de Lusignan S, Dorward J, Correa A, Jones N, Akinyemi O, Amirthalingam $\mathrm{G}$, et al. Risk factors for SARS-CoV-2 among patients in the Oxford Royal College of General Practitioners Research and Surveillance Centre primary care network: a crosssectional study. Lancet Infect Dis. 2020. https://doi.org/10.1016/ S1473-3099(20)30371-6.

22. Palaiodimos L, Kokkinidis DG, Li W, Karamanis D, Ognibene J, Arora S, et al. Severe obesity, increasing age and male sex are independently associated with worse in-hospital outcomes, and higher in-hospital mortality, in a cohort of patients with COVID19 in the Bronx, New York. Metabolism. 2020. https://doi.org/10. 1016/j.metabol.2020.154262.

23. Cecconi M, Piovani D, Brunetta E, Aghemo A, Greco M, Ciccarelli M, et al. Early Predictors of Clinical Deterioration in a Cohort of 239 Patients Hospitalized for Covid-19 Infection in Lombardy, Italy. J Clin Med. 2020. https://doi.org/10.3390/ jcm9051548.

24. Wang L, He W, Yu X, Hu D, Bao M, Liu H, et al. Coronavirus disease 2019 in elderly patients: characteristics and prognostic factors based on 4-week follow-up. J Infect. 2020;80:639-45.

25. Liu Y, Du X, Chen J, Jin Y, Peng L, Wang HHX, et al. Neutrophil-to-lymphocyte ratio as an independent risk factor for mortality in hospitalized patients with COVID-19. J Infect. 2020. https://doi.org/10.1016/j.jinf.2020.04.002.

26. Onder G, Rezza G, Brusaferro S. Case-fatality rate and characteristics of patients dying in relation to COVID-19 in Italy. JAMA. 2020;323:1775-6.

27. National Health Commission of China. The Novel Coronavirus Pneumonia Diagnosis and Treatment Program (7th version). 2020. http://www.nhc.gov.cn/yzygj/s7652m/202003/a3119144. Accessed 4 Mar 2020.

28. WHO Global. Clinical management of COVID-19 interim guidance. 2020. https://www.who.int/publications/i/item/clinical-ma nagement-of-covid-19. Accessed 27 May 2020.

29. Chen S, Zhang Z, Yang J, Wang J, Zhai X, Bärnighausen T, et al. Fangcang shelter hospitals: a novel concept for responding to public health emergencies. Lancet 2020;395:1305-14.

30. Zhang J, Zhou L, Yang Y, Peng W, Wang W, Chen X. Therapeutic and triage strategies for 2019 novel coronavirus disease in fever clinics. Lancet Respir Med. 2020. https://doi.org/10.1016/ S2213-2600(20)30071-0.

31. Yang X, Yu Y, Xu J, Shu H, Xia J, Liu H, et al. Clinical course and outcomes of critically ill patients with SARS-CoV-2 pneumonia in Wuhan, China: a single-centered, retrospective, observational study. Lancet Respir Med. 2020;8:475-81.

32. Zhou F, Yu T, Du R, Fan G, Liu Y, Liu Z, et al. Clinical course and risk factors for mortality of adult inpatients with COVID-19 in Wuhan, China: a retrospective cohort study. Lancet 2020;395: 1054-62.

33. Lameire NH, Bagga A, Cruz D, De Maeseneer J, Endre Z, Kellum JA, et al. Acute kidney injury: an increasing global concern. Lancet 2013;382:170-9.

34. Chen L, Yu J, He W, Chen L, Yuan G, Dong F et al. Risk factors for death in 1859 subjects with COVID-19. Leukemia. 2020. https://doi.org/10.1038/s41375-020-0911-0.

35. Li Q, Cao Y, Chen L, Wu D, Yu J, Wang H, et al. Hematological features of persons with COVID-19. Leukemia. 2020. https://doi. org/10.1038/s41375-020-0910-1. 